\title{
Evaluation of injectable hyaluronic acid-based hydrogels for endodontic tissue regeneration
}

\author{
Esteban Astudillo-Ortiz ${ }^{1,2,3}$, Pedro S Babo ${ }^{1,2}$, Rui L Reis ${ }^{1,2}$ and Manuela E Gomes ${ }^{1,2, *}$
}

1 3B's Research Group, I3Bs - Research Institute on Biomaterials, Biodegradables and Biomimetics, University of Minho, Headquarters of the European Institute of Excellence on Tissue Engineering and Regenerative Medicine, AvePark, Parque de Ciência e Tecnologia, Zona Industrial da Gandra, 4805-017 Barco, Guimarães, Portugal; info@i3bs.uminho.pt

2 ICVS/3B's - PT Government Associate Laboratory, Braga/Guimarães 4805-017, Portugal

3 Department of Endodontics, Area of Histology, School of Dentistry, University of Cuenca, Ecuador; posgrados.odonto@ucuenca.edu.ec

* Correspondence: e-mail@e-mail.com; Tel.: (optional; include country code; if there are multiple corresponding authors, add author initials)

\begin{abstract}
Dental pulp tissue engineering (TE) quests to regenerate dentin/pulp complex by combining a suitable supporting matrix, stem cells, and biochemical stimuli. Such procedures foresee a matrix that can be easily introduced into the root canal system (RCS) and tightly adhere to dentin walls to assure the dentin surface's proper colonization with progenitor cells capable of restoring the dentin/pulp complex. Herein was investigated an injectable self-setting hyaluronic acid-based (HA) hydrogel system, formed by aldehyde-modified (a-HA) with hydrazide-modified (ADH), enriched with platelet lysate (PL), for endodontic regeneration. The hydrogels' working (wT) and setting (sT) times, the adhesion to the dentine walls, the hydrogel's microstructure, and the delivery of human Dental Pulp Cells (DPCs) were studied in vitro. Hydrogels incorporating PL showed a suitable $\mathrm{wT}$ and $\mathrm{sT}$ and a porous microstructure. The tensile tests showed that the breaking point occurs after $4.13 \mathrm{~mm}$ deformation. While in the indentation test after $1.3 \mathrm{~mm}$ deformation. Both breaking points occur in the hydrogel extension. The HA/PL hydrogels exhibited supportive properties and promoted cell migration toward dentin surfaces in vitro. Overall, these results support using PLladen HA injectable hydrogels (HA/PL) as a biomaterial for DPCs encapsulation, thereby displaying great clinical potential towards endodontic regenerative therapies.
\end{abstract}

Keywords: Hyaluronic acid, hydrogels, injectable, tissue engineering, endodontic tissue regeneration.

\section{Introduction}

About $13 \%$ of worldwide population has been subjected to some kind of endodontic treatment on at least one tooth [1,2]. However, the common root canal treatment turns the tooth non-vital. Given the absence of physiological homeostasis-maintenance mechanisms, non-vital teeth present a high risk of long-term failure [3,4]. After root canal treatment, the risk of tooth loss has been s calculated to be around $10 \%$ after five years and close to $20 \%$ at ten years $[5,6]$.

Consequently, during the last decades, therapies capable of maintaining tooth vitality, such as bleeding induction, or capable of regenerating endodontic tissues through tissue-engineered (TE) grafts have been proposed [7]. TE approaches procure to regenerate lost endodontic structures using biomaterials laden with biochemical cues and/or cells. Furthermore, they should enable the anastomosis with hosts tissues and promote the recruitment of suitable progenitor cells allowing the simultaneous regeneration of the pulp and the dentin-pulp complex [8,9].

Hyaluronic acid (HA), has been explored for endodontic TE for its biological and physicochemical features. HA is one of the major structural glycosaminoglycan 
components of the dental pulp extracellular matrix (ECM), and plays an essential role in several cellular tissue functions. It is particularly interesting for TE applications because of its good biocompatibility, gel-forming properties, natural degradation, and ease to modify through its carboxyl and hydroxyl groups in order to produce stable hydrogels $[10,11]$. In particular, the HA hydrogels incorporating platelet lysate (PL) have been shown to support the growth and differentiation of DPCs [12,13]. The addition of hemoderivatives such as platelet lysate (PL) provides the hydrogel with an autologous source of growth factors and fibrin matrices necessary during the early stages of wound healing and tissue regeneration including pulp tissue [14].

Therefore, herein we will evaluate an injectable self-setting hyaluronic acid-based hydrogel enriched with platelet lysate (HAPL) as a hydrogel for regeneration in endodontics. This hydrogel is formed by the spontaneous reaction between the aldehydes from aldehyde-functionalized hyaluronic acid (a-HA) and the hydrazides of adipic acid -hydrazide-modified HA (ADH-HA). The a-HA can further react with primary amines forming Schiff's base bounds, e.g. with the amines of the dentin extracellular matrix (ECM) [15], which would assure a stable linking to the root canal walls fostering an effective cell colonization of the dentin.

\section{Materials and Methods}

\subsection{Preparation of hydrogels precursors}

The hydrogel precursors a-HA and ADH-HA were produced using standard protocols established by our group, as described elsewhere [12].

\subsubsection{Hydrazide-modified Hyaluronic Acid (ADH-HA)}

ADH-HA was produced by modifying the carboxylic acid moieties of the D-glucuronic acid with adipic acid dihydrazide. Adipic acid dihydrazide (ADH) ( $\geq 98 \%$; SigmaAldrich, Saint Louis, USA) was added to a 1 wt.\% HA (Mw 1.5-1.8 MDa; Sigma-Aldrich, Saint Louis, USA) solution prepared in Milli-Q water, at a molar ratio of 1 (ADH:HA). Then, the reaction occurred in the presence of N-(3-Dimethylaminopropyl)-N'-ethylcarbodiimide hydrochloride ( $\geq 98.0 \%$ Sigma-Aldrich, Saint Louis, USA) (molar ratio 0.25; EDCI:HA) at $\mathrm{pH} 4.75$, by addition of $\mathrm{HCl}$ dropwise, at room temperature, until no further $\mathrm{pH}$ variation was observed. The solution was then dialyzed firstly against diluted $\mathrm{HCl}$ containing $0.1 \mathrm{M} \mathrm{NaCl}(3 \times 5 \mathrm{~L}, 48 \mathrm{~h})$, followed by dialysis in diluted $\mathrm{HCl}(\mathrm{pH} 3.5)(3 \times 5$ $\mathrm{L}, 24 \mathrm{~h}$ ) and finally, against Milli-Q water. The resultant solution was frozen at $-80{ }^{\circ} \mathrm{C}$, recovered by freeze-drying, and stored until further use at $-20^{\circ} \mathrm{C}$ [12].

\subsubsection{Aldehyde-modified HA (a-HA)}

The a-HA was produced by sodium periodate (NaIO4) oxidation, as previously described [12]. In summary, HA ( $2.5 \mathrm{mmol}$; Sigma-Aldrich, Saint Louis, USA) was dissolved in mili-Q water at $2 \%$ and an aqueous solution of sodium periodate $(0.5 \mathrm{M}, 5 \mathrm{~mL})$ was added dropwise, stirring for two hours at room temperature in the dark. The reaction was stopped by adding ethylene glycol, and the mixture was transferred into a dialysis membrane (cut-off $14 \mathrm{MDa}$; Sigma-Aldrich, Saint Louis, USA) and purified by dialysis against ultrapure water for three days. Finally, the dried a-HA product was obtained by freezedrying and stored until further use at $-20^{\circ} \mathrm{C}$ and protected from light.

\subsubsection{Platelet lysate (PL)}

PL was prepared from human platelet concentrate by freeze/thaw cycles, using standard protocols, as previously described [16]. A set of 12 batches of platelet concentrate was subjected to three freeze / thaw cycles $\left(-196\right.$ and $\left.37^{\circ} \mathrm{C}\right)$, aliquoted, and stored at -80 ${ }^{\circ} \mathrm{C}$ until needed. Just before use, vials were defrosted in a $37^{\circ} \mathrm{C}$ water bath. Then, the cellular debris was removed by centrifugation at $4000 \mathrm{xg}$ for 5 minutes, and the supernatant was filtered through $0.45 \mu \mathrm{m}$ filters inside a flow chamber. 


\subsubsection{Preparation of PL-laden HA hydrogels (HAPL)}

Hydrogel precursor solutions were prepared at room temperature by dissolving aHA with PBS at $2 \%(w / v)$ (solution A) and ADH-HA in PL also at $2 \%(w / v)$ (solution B). As a control group, a solution of ADH-HA was mixed with PBS (solution C). After well homogenized, the air bubbles were removed by short spinning the vials. Equal volumes of the precursor solution A and B were loaded in a double-barrel syringe, fitted with a static mixer placed at the outlet to produce the injectable HAPL. Whereas equal volumes of A and C solution were loaded in the double-barrel syringe to produce the plain hydrogels (Control).

\subsection{Characterization of hydrogels}

\subsubsection{Working time and setting time}

The working and setting times of plain and PL-laden HA hydrogels were tested as previously described [12]. In brief, a $0.197 \times 0.394$ inch (diameter $x$ length) magnetic stir bar was placed rotating at $100 \mathrm{rpm}$ into the center of a glass petri dish at room temperature (Fig. 1). Then, a $100 \mu \mathrm{L}$ drop of ADH-HA solution in PBS or PL was injected over the magnetic stirrer. The crosslinking reaction was started by adding a $100 \mu \mathrm{L}$ drop of a-HA solution. The working time was counted from the moment at which the precursors were mixed to the gel state, determined when a big lump starts to form from the mixed solutions. The setting time was determined when a solid globule that did not wet the Petri dish's surface was formed. The assay was performed five times for each condition (Fig 1). The process was recorded using a 64 megapixels digital camera (Redmi note 8 pro, Xiaomi, Beijing, China) mounted at $10 \mathrm{~cm}$ from the samples. One previously trained observer was responsible for registering the data.

\subsection{Preparation of tooth slice samples}

Tooth samples were prepared from human teeth, following an established procedure with some modifications $[17,18]$. Briefly, residual soft tissues were removed with a scalpel, and the dental surfaces were wiped down with $70 \%$ ethanol. Next, the teeth were transversally cut with a NTI Superflex diamond disc (Kerr, Orange, California), properly cooled to obtain slices of approximately 2 -mm-thickness. The teeth slices were washed with PBS immediately after cutting.

To the mechanical tensile tests, $5 \mathrm{~mm}$ diameter dentin discs from the coronal region were polished using very fine grit sandpaper (3M, St Paul, Minnesota USA) to obtain flat and smooth surfaces which were then cyanoacrylate bonded to the heads of 10 screws. The dentin discs' opposite surface was also polished until they obtained a flat surface perpendicular to the large axis of the screw.

For the mechanical indentation assays and tooth slice organ cultures, the hole corresponding to the pulp chamber or root canal in the dentin slices of the cervical region was prepared, and standardized using a $1.2 \mathrm{~mm}$ high-speed straight cylinder diamond bur (Kerr, Orange, California), allowing a volume canal ranging between $29-43 \mathrm{~mm}^{3}$.

All the dentin samples were treated following the protocol reported for Aksel in 2020 with some modifications (Aksel et al., 2020). In summary, teeth slices were immersed in 1) $5 \%$ sodium hypochlorite for 2 minutes; 2 ) rinsed with $3 \mathrm{~mL}$ of milli-Q water; 3 ) EDTA $17 \%$ for 5 minutes; 4 ) rinsed with $3 \mathrm{~mL}$ of milli-Q water; 5 ) after that, the surfaces were conditioned with a-HA for 5 minutes; 6) finally, washed with PBS before injecting the hydrogels.

\subsection{Assess the adhesion of HAPL to the dentine walls}

\subsubsection{Scanning Electron Microscopy (SEM) analysis}

HAPL 's interaction with the dentinal walls was analyzed under Scanning Electron Microscopy (SEM). HAPL hydrogels were injected into simulated root canals of dentin discs and set at $37^{\circ} \mathrm{C}$ for 10 mins. Then, the samples were stored at $-20^{\circ} \mathrm{C}$ for 24 hours 
before being transferred to a $-80^{\circ} \mathrm{C}$ freezer for 48 hours. Next, samples were submitted to a freeze-drying process for 48 hours. Finally, each sample was mounted in an aluminum pin and the inner part of the hydrogel was exposed using a scalpel blade to cut the outermost layer, before coating with $\mathrm{Au} / \mathrm{Pd}$ by sputtering for 40 seconds and with a $12 \mathrm{~mA}$ current. The samples were analyzed in a High-Resolution Field Emission Scanning Electron Microscope with Focused Ion Beam-Auriga Compact-Zeiss (Carl Zeiss Microscopy Gmbh, Oberkochen, Germany).

\subsubsection{Tensile test}

After completing the preconditioning step, the dentin discs surfaces were placed in direct contact, while on the opposite side, the screws were held with the clamps of the Instron machine (Instron, Buckinghamshire, UK). Using the Bluehill Universal software, the samples were separated $1 \mathrm{~mm}$ to inject the respective hydrogel using a double-barrel syringe until the surfaces were covered entirely (Fig 3). The distance between the samples was maintained until the setting time of the different solutions was completed. The control hydrogel was allowed to polymerize for 3 minutes and the HAPL for 8 minutes, both at room temperature. In the Bluehill software, the tension method was used, the irregular geometry field was configured, indicating a length of $5 \mathrm{~mm}$ and a rate of $5 \mathrm{~mm}$ per minute (Fig 3). The assays started marking zero-point when the Instron machine registered the first value of tensile forces to standardize the measurements. Maximum load at breakpoint was recorded for each specimen.

\subsubsection{Indentation test}

The dentin samples with the simulated root canal were glued with cyanoacrylate between two metal washers which allowed to stabilize the piece in the support clamps of the Instron machine. Before testing, all simulated root canals of the dentin samples were treated in pre-conditioned as above described. Once the pre-conditioning was complete, polydimethylsiloxane (PDMS) caps were placed in direct contact with the dentin surface of the bottom end of the simulated canal (Fig 4). Using the double-barrel syringe, approximately $100 \mu \mathrm{L}$ of the corresponding hydrogel solution was injected into the simulated root canal, and a PDMS cap was placed in the top end of the simulated canal, and the hydrogel was let to set, to create smooth parallel surfaces. Then, the simulated root canals filled with the respective polymerized hydrogel were loaded immediately into the lower clamp of the Instron machine.

In the Instron machine, through the Bluehill Universal software, the compression test mode was used. A $1 \mathrm{~mm}$ diameter metal pin with a flat end was placed in the upper clamp in direct contact with the hydrogel with a trajectory directed towards the simulated root canal. Zero-point was registred when the Instron machine indicated the first contact between the metal stem and the hydrogel surface, and the last score corresponds to maximum load at break-point for each specimen (Fig 4).

\subsection{Evaluation of hydrogel's ability to deliver stem cells endodontically}

\subsubsection{Expansion of human dental tissues derived cells}

Human dental pulp cells (DPCs) were isolated from human third molars under the scope of previous studies [13]. Both of them were cultured in $75 \mathrm{~cm}^{3} \mathrm{TCP}$ flasks with Dulbecco's Modified Eagle's Medium-low glucose (DMEM, Sigma- Aldrich, St. Louis, USA) supplemented with $10 \%$ fetal bovine serum (FBS) (ThermoFisher Scientific, Waltham, USA) and $1 \%$ antibiotic/antimycotic (ThermoFisher Scientific, Waltham, USA). The culture medium was replaced twice a week and the cultures maintained semi-confluent. All cultures were incubated at $37{ }^{\circ} \mathrm{C}$ in a $5 \% \mathrm{CO} 2$ high-humidity environment. Cells were used between passages 3 and 5 in this study. 
The preparation of the hydrogels was performed similarly to the procedure described in Section 2.1 but under sterile conditions. Briefly, a-HA was dissolved in DPBS, while ADH-HA was dissolved in PL, both at a concentration of $2 \mathrm{wt} \%$ overnight at room temperature. Then, the solutions were sterilized by UV irradiation $(254 \mathrm{~nm})$ for $30 \mathrm{~min}$ prior to cell encapsulation. DPCs cultures were trypsinized and resuspended in culture media, counted, and centrifuged at $300 \mathrm{xg}$ for $5 \mathrm{~min}, 22{ }^{\circ} \mathrm{C}$. The supernatant medium was discarded and $8 \times 106$ cells per $\mathrm{mL}$ were resuspended in the respective ADH-HA/PL solution mixing up and down with a piston pipete. The cell mixture was added to barrel $\mathrm{A}$ of the double-barrel syringe, and barrel B was filled with a-HA (Fig 5).

\subsubsection{Tooth slice organ cultures}

After disinfected and pre-conditioned, simulated root canals $1.2 \mathrm{~mm}$ diameter and 2 $\mathrm{mm}$ of height the dentin slices were filled carefully with approximately $50 \mu \mathrm{L}$ of the hydrogel laden with the corresponding cells and incubated at $37^{\circ} \mathrm{C}$ for $30 \mathrm{~min}$ to form a solid gel (Fig 5). The tooth slice organs (TSOs) of each cell group were cultured in 24-well plates, 3 in basal media, and 3 in osteogenic media for 7 , and 14 days $\left(37^{\circ} \mathrm{C}, 5 \% \mathrm{CO} 2\right)$, changing the culture media twice a week. The TSOs were evaluated during the whole assay by inverted microscopy. Subsequently, after culture time points, the TSOs were fixed in $10 \%$ formalin for $48 \mathrm{~h}$ and analyzed by fluorescence, histochemical and immunohistochemical microscopy.

\subsubsection{Fluorescence microscopy}

After 7 and 14 days in culture, TSOs were washed carefully with PBS before and after fixation with $10 \%(\mathrm{v} / \mathrm{v})$ neutral buffered formalin (ThermoFisher Scientific, Waltham, USA) for $30 \mathrm{~min}$ at room temperature. After, the samples were incubated in a solution of $0.2 \%(\mathrm{v} / \mathrm{v})$ Triton X-100 (Sigma-Aldrich, St. Louis USA) in PBS for 1 hour at room temperature with slight movements. Then, samples were incubated in $1 \mathrm{~mL}$ of the PBS solution containing 4,6-diamidino-2-phenyindole dilactate (DAPI, Biotium, San Francisco,USA) 1:10000 v/v Phalloidin (Phalloidin-Tetramethylrhodamine B isothiocyanate from Amanita phalloides Sigma-Aldrich, St. Louis, USA) 1:200 v/v, for $1 \mathrm{~h}$ at room temperature, under mild agitation. Subsequently, the samples were washed 3 times in PBS for five minutes to reduce the background fluorescence. Then, the samples were visualized under a Fluorescence Inverted Microscope (Zeiss, Axio Observer Z1, Göttingen, Germany), and representative micrographs were taken.

\subsubsection{Histological processing}

After fluorescence analysis, the TSOs were fixed again by 48 hours and demineralized using a decalcifying agent composed of $22,5 \%$ formic acid and $10 \%(\mathrm{w} / \mathrm{v})$ sodium citrate for 2 weeks. The demineralization was confirmed chemically, using a standard protocol [19]. The test was performed daily, mixing $0.5 \mathrm{~mL}$ of the decalcification solution supernatant with $1.0 \mathrm{~mL}$ of citrate-phosphate buffer, and $2.5 \mathrm{~mL}$ of saturated ammonium oxalate solution into a falcon tube. After 20 minutes, a calcium precipitate is formed if the decalcification is still occurring. If positive the tooth samples were washed with distilled water and immersed in fresh decalcifying solution. Else, the test was repeated the following day without refreshing the decalcifying solution. The end-point was considered when the test screened negative results by two consecutive days.

Then, the samples were processed and embedded in paraffin wax for histological and immunohistochemical examination. Sections were cut at $4 \mu \mathrm{m}$ and routinely stained with hematoxylin and eosin.

\subsection{Statistical analysis}

Data were processed using Microsoft Office Excel 2019 and analyzed using GraphPad PRISM 7 (GraphPad Software Inc., CA, USA). Friedman's test was used to detect differences across the measures, followed by an unpaired t-test with Welch's 
correction to find differences between the group samples. Results were presented as mean \pm standard error of the mean. Statistical significance and associated degree of confidence $(\mathrm{p}<0.05)$ are represented by symbols stated in the graphs.

\section{Results}

\subsection{Working time (wT) and setting time (sT)}

The measurements of the apparent in situ gelation time of both hydrogel formulations were summarized in Table 1, described as working and setting times. The control hydrogel started its polymerization process at $10 \mathrm{~s}( \pm 1.4 \mathrm{~s})$ after mixing the precursors and finished after $20 \mathrm{~s}( \pm 1.6 \mathrm{~s})$ (Table 1). The incorporation of PL significantly increased the wT to $2 \mathrm{~min}$ and $18( \pm 29)$, and the sT increased to $6 \mathrm{~min}$ and $13 \mathrm{~s}( \pm 89 \mathrm{~s})(\mathrm{P}=0,0001)$ (Fig 1).

(a)

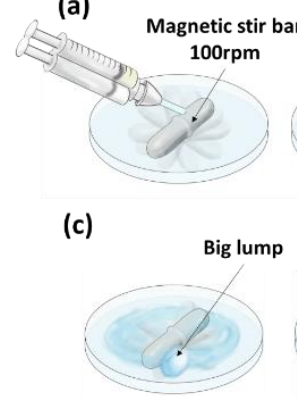

(b)

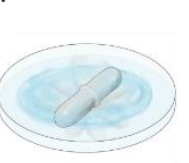

(d)

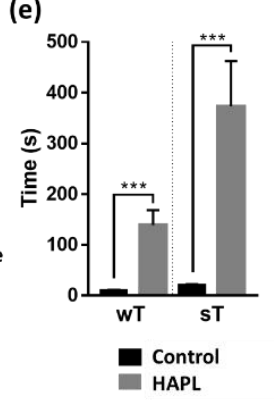

Figure 1. Working and setting time. (a) Injection of the hydrogel precursors directly above the magnetic stir bar. (b) As in the previous stage, the mixed solution keeps liquid flowing as the magnetic stir bar rotates. (c) Working time (wT) was registered when the first lump outline appeared, and the magnetic stir bar showed a slight impediment to turning. (d) Setting time (sT) was registred when the hydrogel formed a solid globule that prevented the normal rotation of the magnetic bar (e) Graphic representation of the wT and sT obtained for the control and HAPL hydrogels.Values represent the mean \pm SD of 5 tests. Symbols $\left(^{* * *}\right)$ denote study groups with statistical significant difference $\mathrm{p}<0,0001$.

Table 1. Descriptive statistics. Time in seconds (s) corresponding to the hydrogels working and setting time.

\begin{tabular}{llrrrrr}
\hline Material & Test & N & Mean $(\mathbf{s})$ & SD $(\mathbf{s})$ & Min $(\mathbf{s})$ & Max (s) \\
\hline Control & wT & 5 & 10.0 & 1.4 & 8 & 12 \\
HAPL & wT & 5 & 138.8 & 29.6 & 105 & 178 \\
Control & sT & 5 & 20.8 & 1.6 & 19 & 23 \\
HAPL & sT & 5 & 373.4 & 89.1 & 302 & 516 \\
\hline
\end{tabular}

\subsection{Dentin pre-conditioning and hydrogel microstructure}

Before pre-conditioning, it was possible to observe that the collagen fibers were exposed and the dentin surfaces covered by debris and smear layer (Fig $2 a$ and $2 b$ ). After pre-conditioning, less debris was present while the collagen fibers were dipped by the aHa layer (Fig 2c and 2d). The HAPL hydrogels presented a heterogeneous, interconnected porous structure, in which larger pores could be observed in the central part, surrounded by smaller pores in the periphery (Fig 2e). When analyzing the hydrogel-dentin interface through SEM, using a magnification of 100x, it was possible to see a direct contact between both (Fig 2f). Analyzing at higher magnifications (500x) was visible a continuum between hydrogel and dentin matrix, and the hydrogel penetration into the dentin tubules present in the walls of the simulated root canal (Fig 2g). 


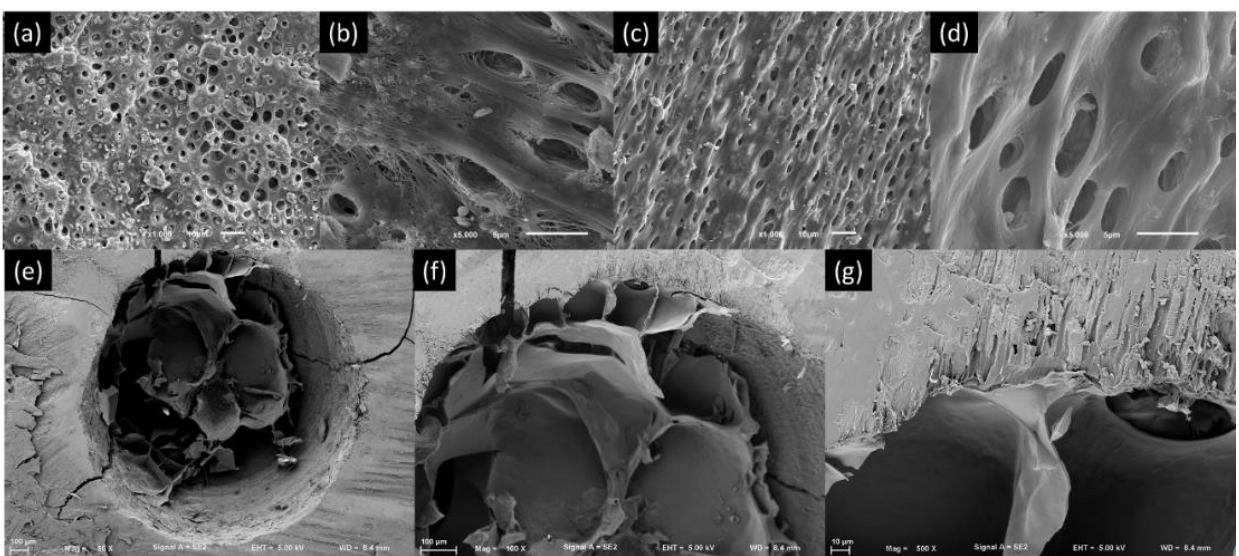

Figure 2. Hydrogel microstructure and interaction with dentin. Representative (a) Dentin of the simulated root canals before the pre-conditioning observed under SEM at 1000x and (b) 5000x. (c) The same simulated root canal after pre-conditioning 1000x and (d) 5000x. (e) Simulated root canal filled with HAPL observed under SEM at 50x, (b) 100x, and (c) 500x. This is a figure. Schemes follow the same formatting.

\subsection{Ability of the hydrogels to adhere to the dentine walls}

After the tensile test, no hydrogel sample was detached from the dentin surfaces. The breaking point was always located in the extension of the hydrogel (Fig 3g). The control hydrogel withstood a displacement of $0.9 \pm 0.1 \mathrm{~mm}$ and tensile stress of $5.6 \pm 2.0 \mathrm{kPa}$ before the break-point (Fig 3h; Table 2). In contrast, the HAPL resisted a $4.3 \pm 1.9 \mathrm{~mm}$ displacement and tensile stress of $0.7 \pm 0.2 \mathrm{kPa}$ (Fig 3i; Table 2).

Likewise, no hydrogel samples were detached from the dentin after the indentation test. In all the assays, the metal stem hole through the hydrogel without compromising the attachment to the dentin (Fig 4h). Before breakpoint, the control hydrogel supported a displacement of $0.269 \pm 0.038 \mathrm{~mm}$ and compressive stress of $0.004 \pm 0.001 \mathrm{kPA}$ (Fig 4i; Table 2). While, after adding PL to the hydrogel, its resistance to displacement was $1.336 \pm 0.306$ $\mathrm{mm}$ and supported compressive stress of $5.096 \pm 1.405 \mathrm{kPa}$ before the break-point (Fig 4j; Table 2).

(a)

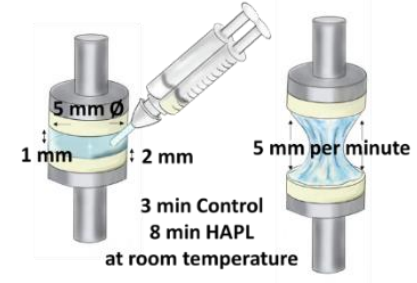

(h)

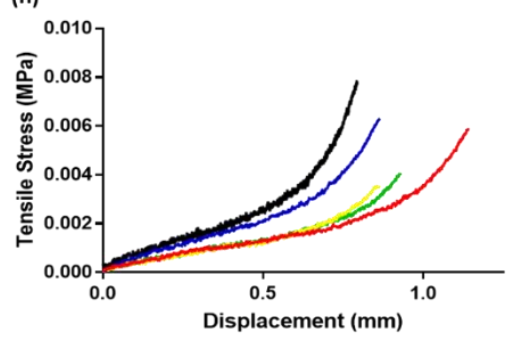

(c)

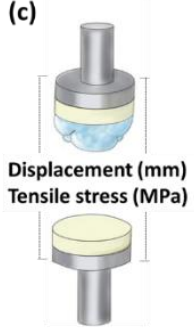

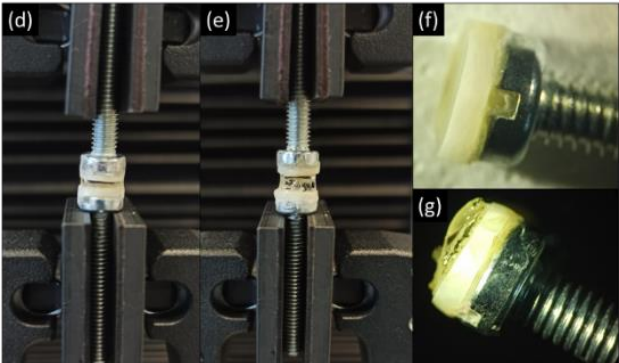

(i)
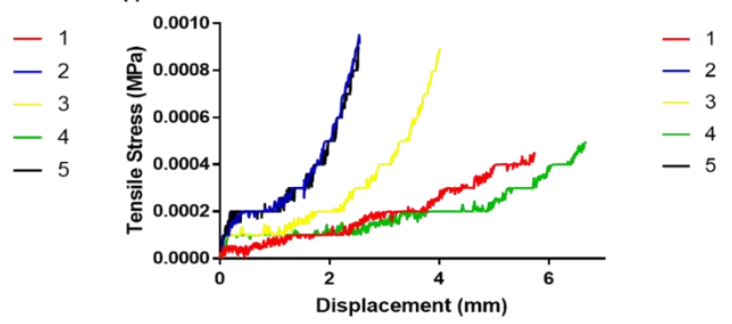

Figure 3. Tensile test. Schematic representation of the tensile test. (a) Loading of hydrogel in the $1 \mathrm{~mm}$ gap between the pre-conditioned dentin disks and zero-point position. (b) Dentin discs were continuously separated at a $5 \mathrm{~mm}$ per minute rate. (c) Breaking point values were registered after hydrogel loss of integrity or detachment from dentine. (d) Dentin disc glued to a screw head before the tensile test, and (e) Sample prepared at the zero-point position, and (f) tensile test ongoing. (g) the hydrogel sample still adhered to the dentin disc after reaching the break-point in the tensile test. (h) Tensile test graphic results of control hydrogel, and (i) HAPL. Each line represents the tensile stress (MPa) curve of one individual sample. 


\subsection{Hydrogel's ability to deliver dental derived cells}

One of the most important hydrogel characteristics for endodontic TE is the ability to deliver stem cells, support their proliferation and colonization of dentin surface, aiming the formation of a dentin/pulp complex-like tissue.

In this assay, HAPL remained stable inside the tooth slice organ (TSO), however presenting signs of degradation when removed from wells to be processed. For extended culturing times (day 14), the hydrogel almost entirely degraded and was replaced by dental pulp-like tissues that were attached to the walls of the simulated root canal

The presence of cells after each timepoint was confirmed through DAPI-phalloidin staining using fluorescence microscopy. The immunofluorescence analysis showed that the DPCs presented a typical spindle-like shape during the first seven days and allowed migration of DPCs towards the dentin walls (Fig 5e). From day ten on, the hydrogel was degraded centrifugally, and it was possible to observe that the DPCs gradually converged towards the dentin walls, forming conglomerates.

Histologically, after 7 days, it was possible to observe cells approaching the dentin surface, depositing an incipient ECM. On day 14, the histological analysis showed the deposition of a loose-type extracellular matrix composed of few fibers and abundant ground substance derived from DPCs near dentinal walls.

(a)

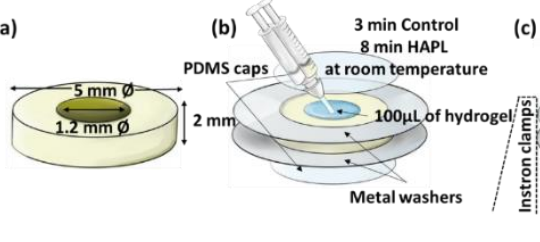

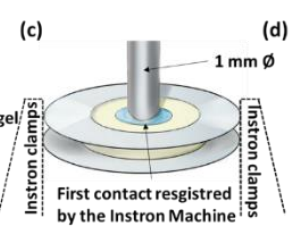

(d)

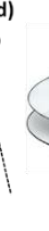

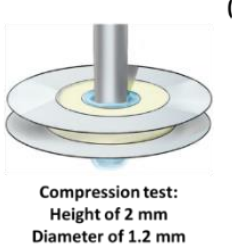

(e)

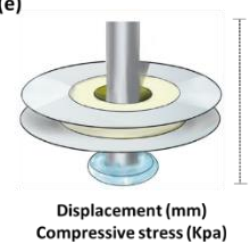

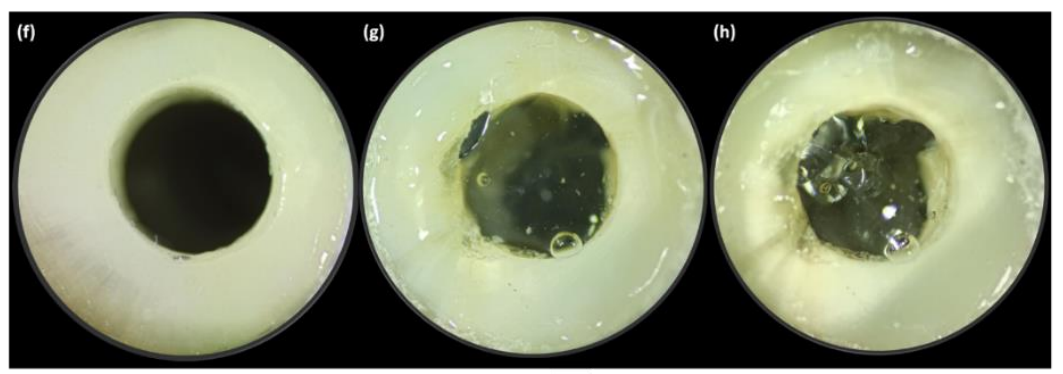

(i)
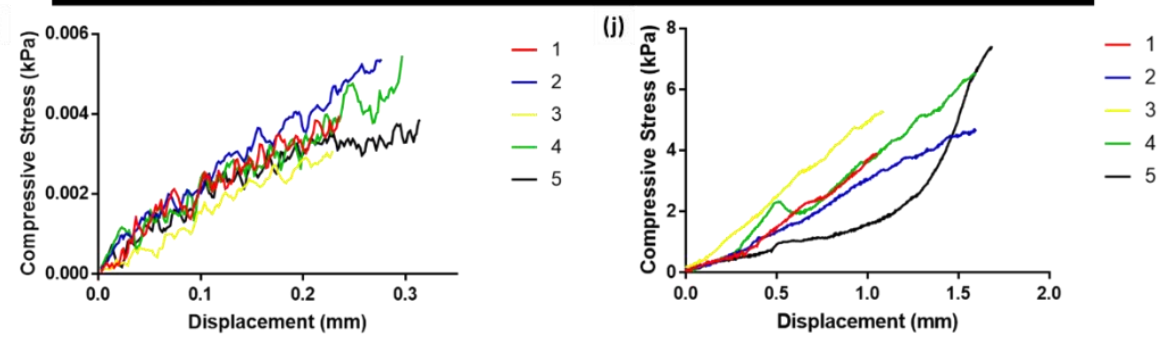

Figure 4. Indentation test. (a) Dentin disc obtained from a sound tooth (b) Sample preparation. (c) Dentin disc mounted on the Instron machine before starting the test. (d) The metal stem is introduced at a continuous rate into the simulated canal filled with the hydrogel. (e) The breakingpoint corresponding to hydrogel's loss of integrity or detachment from the dentin walls. (f) Pre-conditioned dentin disk before injection of the hydrogel. (g) Simulated root canal filled with HAPL, ready to be used on the indentation test. (h) Same sample after the indentation test. (i) Indentation test graphic of control hydrogel or (j) HAPL. Each line represents the compressive stress $(\mathrm{kPa})$ curve or one individual sample.

Table 2. Descriptive statistics. Tensile and indentation tests.

\begin{tabular}{llllllllll}
\hline Test & Material & Measure & $\mathbf{1}$ & $\mathbf{2}$ & $\mathbf{3}$ & $\mathbf{4}$ & $\mathbf{5}$ & Mean & SD \\
\hline Tensile & Control & Displacement $(\mathrm{mm})$ & 1.1412 & 0.8674 & 0.8643 & 0.9301 & 0.7943 & $\mathbf{0 . 9 1 9 4}$ & 0.1329 \\
\hline
\end{tabular}




\begin{tabular}{|c|c|c|c|c|c|c|c|c|c|}
\hline & & Tensile stress (MPa) & 0.0061 & 0.0061 & 0.0043 & 0.0043 & 0.0082 & 0.0058 & 0.0016 \\
\hline & \multirow{2}{*}{ HAPL } & Displacement (mm) & 5.7341 & 2.5421 & 4.0172 & 6.6927 & 2.5673 & 4.3106 & 1.8677 \\
\hline & & Tensile stress (MPa) & 0.0004 & 0.0009 & 0.0009 & 0.0005 & 0.0009 & 0.0007 & 0.0002 \\
\hline \multirow{4}{*}{ Indentation } & \multirow{2}{*}{ Control } & Displacement (mm) & 0.2333 & 0.2815 & 0.2266 & 0.2906 & 0.3142 & 0.2692 & 0.0379 \\
\hline & & Compressive stress $(\mathrm{kPa})$ & 0.0040 & 0.0054 & 0.0031 & 0.0055 & 0.0038 & 0.0043 & 0.0010 \\
\hline & \multirow{2}{*}{ HAPL } & Displacement (mm) & 1.0541 & 1.6003 & 1.0902 & 1.6001 & 1.6834 & 1.4056 & 0.3065 \\
\hline & & Compressive stress $(\mathrm{kPa})$ & 3.9074 & 4.6874 & 5.2862 & 6.5021 & 7.4143 & 5.5594 & 1.4047 \\
\hline
\end{tabular}

\section{Discussion}

This work aimed to evaluate if the injectable aldehyde-hydrazide hyaluronic acidbased hydrogel enriched with platelet lysate encapsulating DPCs has the suitable properties and abilities for its potential use in regenerative endodontics. The analysis performed in this in vitro study allowed us to evaluate the gelation characteristics, injectability, bound to dentin walls, and cell-supportive properties of a class of injectable self-setting aldehyde-hydrazide hyaluronic acid-based hydrogel enriched with platelet lysate as a TE construct aiming endodontic tissue regeneration.

The choice of an injectable hydrogel for endodontic regeneration is based on its similarities to soft tissues, providing an environment physiologically suitable for cell growth. The hydrogels mimic the ECM properties such as high-water content, controllable porosity, and mechanical and physicochemical properties, supporting the natural functions of cells [20]. Specifically, in the case of tuneable aldehyde-hydrazide-based hydrogels is also possible to allow the development of cell morphologies similar to the original tissues [20]. Moreover, the reaction between aldehydes and hydrazides forms Schiff bases capable of generating linkages with the collagen fibers of the dentin [20], enhancing the integration of the hydrogel TE graft into host's tissues.

(a) Tooth preparation

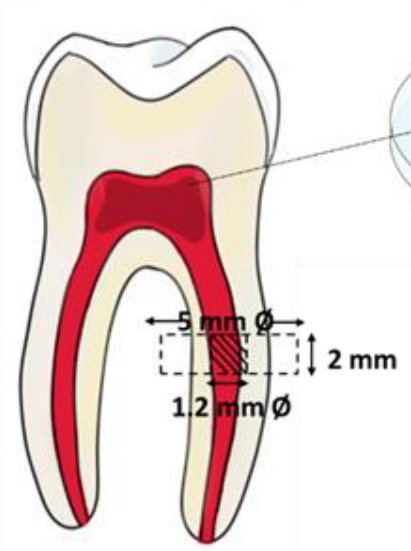

(e)

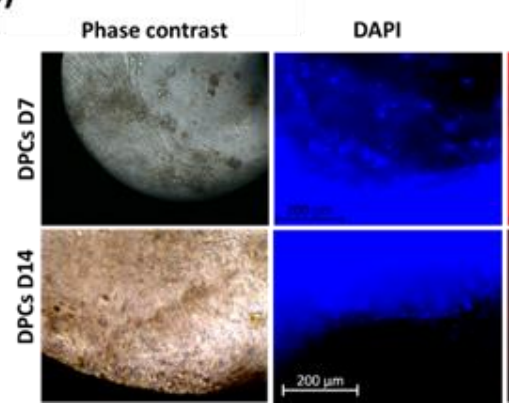

(b) Cell expansion

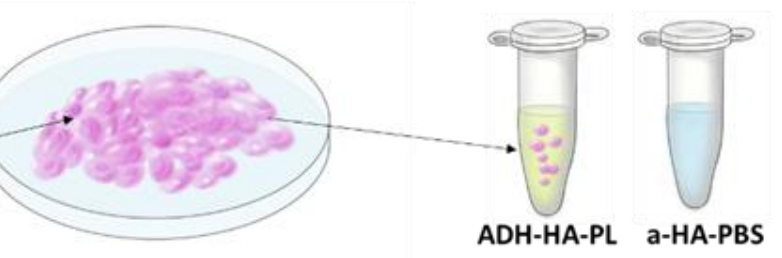

(c) Hydrogel preparation

(d) Simulated root canal filling
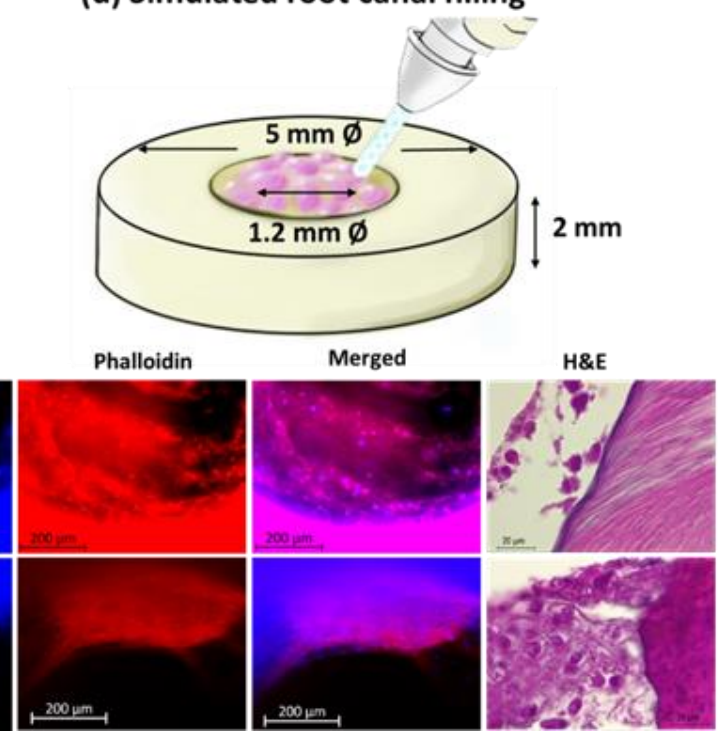
Figure 5. Hydrogels' ability to deliver DPCs. (a) Schematic representative of the preparation of a tooth slice organ for cell culture. A human sound tooth was used to get DPCs. (b) DPCs cells isolated from the human sound tooth were cultured to test the hydrogel's ability to deliver stem cells. (c) $8 \times 106$ of cells per $\mathrm{mL}$ were resuspended in ADH-HA-PL hydrogel precursor solution (d) Using a double-barrel syringe, the hydrogel's precursors were mixed and injected into preconditioned dentin discs of $5 \mathrm{~mm}$ diameter and $2 \mathrm{~mm}$ heigh with a hole standardized at $1.2 \mathrm{~mm}$ diameter corresponding to its root canal were used as tooth slice organ TSO. The TSOs were cultured to test the hydrogel's ability to deliver dental stem cells. DPCs encapsulated in HAPL (e) TSO containing DPCs after 7 days and 14 days in culture by phase-contrast microscopy (10x), fluorescence microscopy (DAPIPhalloidin staining; 20x) and representative histological section (H\&E staining; 100x). Indentation test.

Although the expected therapeutic function of this TE approach is not the same as an endodontic sealer, i.e. its function (aim) is not to seal the root canal but to regenerate the endodontic tissues, we will discuss some characteristics against endodontic sealers due to the similarities in its handling and application. The injectability of these hydrogels is one of the main advantages for endodontic applications, allowing the material to fill root canal systems in all their complexity, emulating a technique often applied in silicon-based sealers [21]. As herein observed, the hydrogel tested can be easily applied into simulated root canals prepared using standard instrumentation and preconditioning protocols. Thus it is fair to assume that it will find familiarity among clinicians. At the same time, its self-setting properties assure that polymerization occurred in the whole injected solution, maintaining the same properties along the entire root canal.

Regarding the working time, the HAPL is faster $(2.18 \pm 0.29 \mathrm{~m})$ than the referred values of established endodontic sealants, such as those studied by Zhou in 2013, including resin-based endodontic sealers such as AH plus $(240 \pm 40 \mathrm{~m})$ and silicone-based sealers such as Guttaflow $(15 \pm 5 \mathrm{~m})$ [21]. However, it is necessary to clarify that the methods used to measure the working time were different. The increase of the working time promoted by incorporating PL could be enough for a future clinical application. So, the consequences of a short working time are related to the lack of flow inside the root canal and the little or no capacity to reach its total length and its anfractuosities [22]. The working time of HAPL is comparable to that of Dycal (2.20 min), a material that was initially designed as an endodontic sealer [23] but was later used as a pulp protector due to its reaction speed. However, Dycal develops its maximum physical properties almost immediately (sT 2.5 to $3.5 \mathrm{~min}$ ), unlike HAPL (6.13 $\mathrm{min}$ ) [24]. Moreover, the swelling characteristic in hydrogels [25] would refill any slight filling deficiency in the canal, complementing it with sonic or ultrasonic activation to enhance fluency and adaptation [26].

The setting time of the HAPL hydrogel $(6.13 \pm 1.29 \mathrm{~m})$ is lower than endodontic sealers as AH plus $(690 \pm 90 \mathrm{~m})$ and Guttaflow $(42 \pm 6 \mathrm{~m})$. However, for a future clinical application, a working time of $6 \mathrm{~m}$ would allow a comfortable application during a clinical session. Moreover, taking into consideration that the canal must be sealed with a bioceramic material to close the top end of the cavity, then with glass ionomer and a composite resin as was used in recent clinical case reports [27,28], the time management is critical for the comfort for the patient.

Concerning the microstructure, the hydrogel presented open pores and interconnections that would facilitate the migration, nutrition, and proliferation of DPCs inside $[29,30]$. These results align with previous studies reported by other authors $[12,13]$. Furthermore, the microstructural analysis of the hydrogel also allowed to observe its penetration into the dentin tubules and its close interaction with the dentin matrix collagen fibers. The pre-conditioning of the dentin surface, as herein performed, allows the tight interaction of the hydrogel with dentin matrix both by 1) transforming the reactive amines of dentin collagen into HA with reactive aldehydes by Schiff's base reaction, thus bridging the reaction of the hydrogel matrix with the protein matrix of the dentin [20,31], and 2) creating a moist and homogeneous surface, facilitating the penetration of the hydrogel into the microtubules. This fact provides the hydrogel non only a chemical but also physical micro-adhesions that reinforce its bond to the dentin surface $[15,32]$. 
The tensile and indentation tests confirmed the above described. The hydrogel samples always remained attached to the dentin in both cases, while the breaking point was located within the hydrogel structure. Therefore, the data obtained explain the resistance values on themselves and assume that the values corresponding to the hydrogel-dentin interface are superior to those obtained. Moreover, the present in vitro study demonstrated that the incorporation of PL increased the resistance to the displacement from 0.9 \pm 0.1 to $4.3 \pm 1.9 \mathrm{~mm}$ at the same time that decreased the resistance to tensile stress from 6 \pm 2 to $0.7 \pm 0.2 \mathrm{kPa}$ in the tensile test using an Instron Machine. In other words, PL incorporation into the HAPL hydrogel raises its flexibility while reduces its resistance to tensile forces, similar to the reported in the existing literature [12,13,33].

In the present study, HAPL was shown to support the delivery of cells to simulated root canals. During fourteen days, the hydrogels fostered the migration towards the dentin walls in vitro. Using phase-contrast microscopy, the cells encapsulated in the hydrogel migrated towards the periphery of the root canal while degrading the hydrogel and forming their matrix (Fig 5f). After H\&E staining, it was possible to confirm the presence of DPCs, which showed signs of migration towards the dentin walls as soon as 1 week after implantation (Fig 5g).

Once there, dentin matrix would encourage DPCs adhesion, proliferation, and differentiation into odontoblast-like cells due to the presence of specific growth factors in its demineralized matrix, as was reported by Huang, Liu, and Salehi [34-36]. However, further investigation must be performed to confirm the latter in three-dimensional culture conditions or an in vivo assay because, in static culture, the nutrients and oxygen cannot flow efficiently throughout the three-dimensional matrices, which causes cell necrosis in the center part of the construct, as was reported by Tayebi [37].

Has previously reported, HA hydrogel formulations similar to the HAPL herein studied degrade quite fast, particularly in the presence of hyaluronidase, an enzyme expressed in inflamed or remodeling tissues [12]. In general, Schiff bases are characterized by poor degradation, but aldehyde-hydrazide-based hydrogels form a subclass of Schiff's base called hydrazone bond which presents excellent ability of hydrolysis in aqueous environments [15]. Therefore, to enhance the stability of the material against degradation, some researchers have reinforced the hydrogel with materials such as graphene or cellulose, obtaining satisfactory results in vitro $[12,16,38,39]$. However, we prefer not to use these reinforcements because it is still unknown whether they could generate foreign body reactions at the periapical zone as the classical cases reported by Koppang and Nair $[40,41]$. Moreover, HAPL degradation was centrifugal in all the samples, which means it began at the level of the pulp core and spread towards the periphery along the time. PLladen HA hydrogels have been shown to entrap angiogenic, chemotactic and morphogenic factors, such as vascular endothelial growth factor (VEGF) and platelet-derived growth factor (PDGF), among others, which are released in a hydrogel degradation-dependent manner [12-14]. This centrifugal degradation triggers the release of chemotactic growth factors that could allow a host tissue invasion carrying blood vessels in the core of the newly formed tissue. This fact gives an option to overcome the issues related to the regeneration of the complex hierarchical distribution present on the dental pulp tissues, i.e. a vascularized and innervated pulp core composed of loose connective tissue surrounded by specific dental cells such as odontoblasts [10].

\section{Conclusions}

The HAPL injectable and self-setting hydrogel provide familiar and straightforward applicability in endodontics. PL incorporation to the aldehyde-hydrazide hyaluronic acidbased hydrogel increases working time and setting time to handier levels allowing a suitable root canal filling. Furthermore, the hydrogel adheres tightly to the dentin matrix and penetrates the dentin tubules. Together with hydrogels resilience to displacement, this material is suitable for the function and the forces it will be subjected. Envisioning TE approaches aiming endodontic regeneration, the hydrogel is suited to deliver DPCs into 
instrumented and conditioned root canals, favored cell migration towards the dentin walls, and after the hydrogel degradation, it was replaced by extracellular matrix derived from DPCs. In a nutshell, HAPL hydrogel is a promissory biomaterial that demonstrates excellent performance needs further investigation for its potential clinical application in regenerative endodontics.

Author Contributions: Esteban Astudillo-Ortiz: Conceptualization, Investigation, Methodology, Formal analysis, Validation, Writing- Original draft preparation; Pedro S. Babo: Conceptualization, Methodology, Investigation, Validation, Supervision, Writing - Review \& Editing; Rui L. Reis: Supervision; Manuela E. Gomes: Supervision, Funding acquisition, Writing - Review \& Editing.

All authors have given approval to the final version of the manuscript

Funding: This research was funded by NORTE2020 (NORTE-01-0145-FEDER-000021) and InjecTEProject ID: 287953, financed by NFR under NANO2021. FCT projects PTDC/NAN-MAT/30595/2017 and PTDC/CTM-CTM/29930/2017. Secretary of Higer Educaton, Science, Technology and Innovation, SENESCYT (Spanish acronym) from Ecuador, reference No. CZ06-000132-2017-01.

Institutional Review Board Statement: All the collected human samples, namely teeth, cells, and blood derivates, and their manipulation were performed following the national and international guidelines. Human teeth were extracted for orthodontic reasons at Service of Oral Surgery of the Malo Clinic Dental Care (Portugal), following the standards of quality and security (as established in 2004/23/CE, 2006/17/CE, and 2006/86/CE), under an institutional board protocol established and approved by the ethical committee for health at 23/12/2014. Platelet concentrate collections, obtained from volunteer donations from healthy donors by 2005/62/CE, were performed at Serviço de ImunoHemoterapia-Centro Hospitalar de São João (Portugal) provided under an approved institutional board protocol (ethical commission of CHSJ/FMUP approved at 18/03/2018). Furthermore, all human donors provided written informed consent before enrolment under the Declaration of Helsinki (59th WMA General Assembly, Seoul, Korea, October 2008).

Informed Consent Statement: Not applicable.

Data Availability Statement: The data used in this study can be accessed from the authors with reasonable requests.

Acknowledgments: Esteban Astudillo-Ortiz acknowledges Emanuel Fernandes's teaching and technical support in the Instron Machine and Bluehill Universal software.

Conflicts of Interest: The authors declare no conflict of interest.

\section{References}

References must be numbered in order of appearance in the text (including citations in tables and legends) and listed individually at the end of the manuscript. We recommend preparing the references with a bibliography software package, such as EndNote, ReferenceManager or Zotero to avoid typing mistakes and duplicated references. Include the digital object identifier (DOI) for all references where available.

Citations and references in the Supplementary Materials are permitted provided that they also appear in the reference list here.

In the text, reference numbers should be placed in square brackets [ ] and placed before the punctuation; for example [1], [1-3] or $[1,3]$. For embedded citations in the text with pagination, use both parentheses and brackets to indicate the reference number and page numbers; for example [5] (p. 10), or [6] (pp. 101-105).

1. Boykin, M. J.; Gilbert, G. H.; Tilashalski, K. R.; Shelton, B. J. Incidence of Endodontic Treatment: A 48-Month Prospective Study. J. Endod. 2003, 29 (12), 806-809. https://doi.org/10.1097/00004770-200312000-00005.

2. Peres, M. A.; Macpherson, L. M. D.; Weyant, R. J.; Daly, B.; Venturelli, R.; Mathur, M. R.; Listl, S.; Celeste, R. K.; GuarnizoHerreño, C. C.; Kearns, C.; Benzian, H.; Allison, P.; Watt, R. G. Oral Diseases: A Global Public Health Challenge. The Lancet. Lancet Publishing Group July 20, 2019, pp 249-260. https://doi.org/10.1016/S0140-6736(19)31146-8.

3. Schmalz, G.; Smith, A. J. Pulp Development, Repair, and Regeneration: Challenges of the Transition from Traditional Dentistry to Biologically Based Therapies. J. Endod. 2014, 40 (4 SUPPL.), S2-S5. https://doi.org/10.1016/j.joen.2014.01.018.

4. Hargreaves, K. M.; Berman, L. H. Cohen's Pathways of the Pulp, 11th ed.; Elsevier: St. Louis, 2016.

5. Fransson, H.; Dawson, V. S.; Frisk, F.; Bjørndal, L.; Jonasson, P.; Kvist, T.; Markvart, M.; Petersson, K.; Pigg, M.; Reit, C.; Wolf, E. Survival of Root-Filled Teeth in the Swedish Adult Population. J. Endod. 2016, 42 (2), 216-220. https://doi.org/10.1016/j.joen.2015.11.008. 
6. Landys Borén, D.; Jonasson, P.; Kvist, T. Long-Term Survival of Endodontically Treated Teeth at a Public Dental Specialist Clinic. J. Endod. 2015, 41 (2), 176-181. https://doi.org/10.1016/j.joen.2014.10.002.

7. Schmalz, G.; Widbiller, M.; Galler, K. M. Clinical Perspectives of Pulp Regeneration. J. Endod. 2020,46 (9), S161-S174. https://doi.org/10.1016/j.joen.2020.06.037.

8. Shrestha, S.; Diogenes, A.; Kishen, A. Temporal-Controlled Release of Bovine Serum Albumin from Chitosan Nanoparticles: Effect on the Regulation of Alkaline Phosphatase Activity in Stem Cells from Apical Papilla. J. Endod. 2014, 40 (9), 1349-1354. https://doi.org/10.1016/i.joen.2014.02.018.

9. Marí-Beffa, M.; Segura-Egea, J. J.; Díaz-Cuenca, A. Regenerative Endodontic Procedures: A Perspective from Stem Cell Niche Biology. J. Endod. 2017, 43 (1), 52-62. https://doi.org/10.1016/j.joen.2016.09.011.

10. Berman, L. H.; Hargreaves, K. M. Cohen's Pathway of The Pulp; Elsevier: St. Louis, Missouri, 2020.

11. Burdick, J. A.; Prestwich, G. D. Hyaluronic Acid Hydrogels for Biomedical Applications. Adv. Mater. 2011,23 (12), 41-56. https://doi.org/10.1002/adma.201003963.

12. Silva, C. R.; Babo, P. S.; Gulino, M.; Costa, L.; Oliveira, J. M.; Silva-Correia, J.; Domingues, R. M. A.; Reis, R. L.; Gomes, M. E. Injectable and Tunable Hyaluronic Acid Hydrogels Releasing Chemotactic and Angiogenic Growth Factors for Endodontic Regeneration. Acta Biomater. 2018, 77, 155-171. https://doi.org/10.1016/j.actbio.2018.07.035.

13. Almeida, L. D. F.; Babo, P. S.; Silva, C. R.; Rodrigues, M. T.; Hebling, J.; Reis, R. L.; Gomes, M. E. Hyaluronic Acid Hydrogels Incorporating Platelet Lysate Enhance Human Pulp Cell Proliferation and Differentiation. J. Mater. Sci. Mater. Med. 2018, 29 (6). https://doi.org/10.1007/s10856-018-6088-7.

14. Babo, P.; Santo, V. E.; Duarte, A. R. C.; Correia, C.; Costa, M. H. G.; Mano, J. F.; Reis, R. L.; Gomes, M. E. Platelet Lysate Membranes as New Autologous Templates for Tissue Engineering Applications. Inflamm. Regen. 2014,34 (1), $033-044$. https://doi.org/10.2492/inflammregen.34.033.

15. Li, S.; Xia, Y.; Qiu, Y.; Chen, X.; Shi, S. Preparation and Property of Starch Nanoparticles Reinforced Aldehyde-Hydrazide Covalently Crosslinked PNIPAM Hydrogels. J. Appl. Polym. Sci. 2018, 135 (5), 1-13. https://doi.org/10.1002/app.45761.

16. Mendes, B. B.; Gómez-Florit, M.; Araújo, A. C.; Prada, J.; Babo, P. S.; Domingues, R. M. A.; Reis, R. L.; Gomes, M. E. Intrinsically Bioactive Cryogels Based on Platelet Lysate Nanocomposites for Hemostasis Applications. Biomacromolecules 2020,21 (9), 3678-3692. https://doi.org/10.1021/acs.biomac.0c00787.

17. Sloan, A. J.; Shelton, R. M.; Hann, A. C.; Moxham, B. J.; Smith, A. J. An in Vitro Approach for the Study of Dentinogenesis by Organ Culture of the Dentine-Pulp Complex from Rat Incisor Teeth. Arch. Oral Biol. 1998, 43 (6), $421-430$. https://doi.org/10.1016/S0003-9969(98)00029-6.

18. Saw, T. Y.; Cao, T.; Yap, A. U. J.; Lee Ng, M. M. Tooth Slice Organ Culture and Established Cell Line Culture Models for Cytotoxicity Assessment of Dental Materials. Toxicol. Vitr. 2005, 19 (1), 145-154. https://doi.org/10.1016/j.tiv.2004.08.006.

19. Barros, V.; Gomes, M. E.; Dias, I. R. Contribution to the Characterization of the Glucocorticoid Treated Ovariectomized Osteoporotic Sheep Model for Pre-Clinical and Translational Studies in Orthopaedic Research- Bone Mineral Density and Histological and Histomorphometric Analysis, Universidade de Trás-os-Montes e Alto Douro, 2018.

20. Xu, J.; Liu, Y.; Hsu, S. hui. Hydrogels Based on Schiff Base Linkages for Biomedical Applications. Molecules 2019, 24 (16), 1-21. https://doi.org/10.3390/molecules24163005.

21. Zhou, H. M.; Shen, Y.; Zheng, W.; Li, L.; Zheng, Y. F.; Haapasalo, M. Physical Properties of 5 Root Canal Sealers. J. Endod. 2013, 39 (10), 1281-1286. https://doi.org/10.1016/j.joen.2013.06.012.

22. Trope, M.; Bunes, A.; Debelian, G. Root Filling Materials and Techniques: Bioceramics a New Hope? Endod. Top. 2015, 32 (1), 86-96. https://doi.org/10.1111/etp.12074.

23. Goldberg, F.; Gurfinkel, J. Analysis of the Use of Dycal with Gutta-Percha Points as an Endodontic Filling Technique. Oral Surgery, Oral Med. Oral Pathol. 1979, 47 (1), 78-82. https://doi.org/10.1016/0030-4220(79)90106-3.

24. Stanley, H. R.; Pameijer, C. Pulp Capping with a New Visible-Light-Curing Calcium Hydroxide Composition (Prisma VLC Dycal). Oper. Dent. 1985, 10, 156-163.

25. Louf, J. F.; Lu, N. B.; O'Connell, M. G.; Cho, H. J.; Datta, S. S. Under Pressure: Hydrogel Swelling in a Granular Medium. Sci. Adv. 2021, 7 (7), 1-11. https://doi.org/10.1126/sciadv.abd2711.

26. Wiesse, P. E. B.; Silva-Sousa, Y. T.; Pereira, R. D.; Estrela, C.; Domingues, L. M.; Pécora, J. D.; Sousa-Neto, M. D. Effect of Ultrasonic and Sonic Activation of Root Canal Sealers on the Push-out Bond Strength and Interfacial Adaptation to Root Canal Dentine. Int. Endod. J. 2018, 51 (1), 102-111. https://doi.org/10.1111/iej.12794.

27. Nakashima, M.; Iohara, K.; Murakami, M.; Nakamura, H.; Sato, Y.; Ariji, Y.; Matsushita, K. Pulp Regeneration by Transplantation of Dental Pulp Stem Cells in Pulpitis: A Pilot Clinical Study. Stem Cell Res. Ther. 2017, 8 (1), 1-13. https://doi.org/10.1186/s13287-017-0506-5.

28. Meza, G.; Urrejola, D.; Saint Jean, N.; Inostroza, C.; López, V.; Khoury, M.; Brizuela, C. Personalized Cell Therapy for Pulpitis Using Autologous Dental Pulp Stem Cells and Leukocyte Platelet-Rich Fibrin: A Case Report. J. Endod. 2019,45 (2), 144-149. https://doi.org/10.1016/j.joen.2018.11.009.

29. Hanson, A. J.; Quinn, M. T. Effect of Fibrin Sealant Composition on Human Neutrophil Chemotaxis. J. Biomed. Mater. Res. 2002, 61 (3), 474-481. https://doi.org/10.1002/jbm.10196.

30. Wang, F. M.; Qiu, K.; Hu, T.; Wan, C. X.; Zhou, X. D.; Gutmann, J. L. Biodegradable Porous Calcium Polyphosphate Scaffolds for the Three-Dimensional Culture of Dental Pulp Cells. Int. Endod. J. 2006, 39 (6), 477-483. https://doi.org/10.1111/j.13652591.2006.01114.x.

31. Perdigão, J. Current Perspectives on Dental Adhesion: (1) Dentin Adhesion - Not There Yet. Jpn. Dent. Sci. Rev. 2020, 56 (1), 190-207. https://doi.org/10.1016/j.jdsr.2020.08.004. 
32. Yang, J.; Bai, R.; Chen, B.; Suo, Z. Hydrogel Adhesion: A Supramolecular Synergy of Chemistry, Topology, and Mechanics. Adv. Funct. Mater. 2020, 30 (2). https://doi.org/10.1002/adfm.201901693.

33. Babo, P. S.; Pires, R. L.; Santos, L.; Franco, A.; Rodrigues, F.; Leonor, I.; Reis, R. L.; Gomes, M. E. Platelet Lysate-Loaded Photocrosslinkable Hyaluronic Acid Hydrogels for Periodontal Endogenous Regenerative Technology. ACS Biomater. Sci. Eng. 2017, 3 (7), 1359-1369. https://doi.org/10.1021/acsbiomaterials.6b00508.

34. Liu, G.; Xu, G.; Gao, Z.; Liu, Z.; Xu, J.; Wang, J.; Zhang, C.; Wang, S. Demineralized Dentin Matrix Induces Odontoblastic Differentiation of Dental Pulp Stem Cells. Cells Tissues Organs 2015, 201 (1), 65-76. https://doi.org/10.1159/000440952.

35. Salehi, S.; Cooper, P.; Smith, A.; Ferracane, J. Dentin Matrix Components Extracted with Phosphoric Acid Enhance Cell Proliferation and Mineralization. Dent. Mater. 2016, 32 (3), 334-342. https://doi.org/10.1016/j.dental.2015.11.004.

36. Huang, G. T. J.; Shagramanova, K.; Chan, S. W. Formation of Odontoblast-Like Cells from Cultured Human Dental Pulp Cells on Dentin In Vitro. J. Endod. 2006, 32 (11), 1066-1073. https://doi.org/10.1016/j.joen.2006.05.009.

37. Tayebi, L. Applications of Biomedical Engineering in Dentistry, 1st ed.; Springer: Cham, 2020. https://doi.org/10.1007/978-3-030$\underline{21583-5}$.

38. Domingues, R. M. A.; Silva, M.; Gershovich, P.; Betta, S.; Babo, P.; Caridade, S. G.; Mano, J. F.; Motta, A.; Reis, R. L.; Gomes, M. E. Development of Injectable Hyaluronic Acid/Cellulose Nanocrystals Bionanocomposite Hydrogels for Tissue Engineering Applications. Bioconjug. Chem. 2015, 26 (8), 1571-1581. https://doi.org/10.1021/acs.bioconjchem.5b00209.

39. Patil, R.; Kansara, V.; Ray, D.; Aswal, V. K.; Jha, P. K.; Bahadur, P.; Tiwari, S. Slow Degrading Hyaluronic Acid Hydrogel Reinforced with Cationized Graphene Nanosheets. Int. J. Biol. Macromol. 2019, 141, 232-239. https://doi.org/10.1016/j.ijbiomac.2019.08.243.

40. Nair, P. N. R. Critical Reviews in Oral Biology \& Medicine and the causes of endodontic failures. Int. Am. Assoc. Dent. Res. 2004, 15 (6), 348-381.

41. Koppang, H. S.; Koppang, R.; Solheim, T.; Aarnes, H.; Stølen, S. Ø. Cellulose Fibers from Endodontic Paper Points as an Etiological Factor in Postendodontic Periapical Granulomas and Cysts. J. Endod. 1989, 15 (8), 369-372. https://doi.org/10.1016/S0099$\underline{2399(89) 80075-5 .}$. 